\title{
Introducing Biofilms
}

\section{W. J. Costerton and M. Wilson}

The concept that bacteria live preferentially in matrixenclosed communities attached to surfaces has emerged gradually from scientific observations over an extended period of time, but the pace at which this concept has advanced has accelerated sharply during the past two decades. Because Antonie van Leuwenhoek examined his own teeth scrapings with his primitive microscope, he probably saw more biofilm fragments than planktonic cells, and dental microbiologists and waste-water engineers have had a lengthy association with biofilms without using that term. Early microscopic observations of marine systems showed that most bacteria adhered actively to surfaces, and the role of surfaces in the migration and maturation of myxobacterial communities was noted very early in the study of these fascinating organisms. The new concept that was crystallized in a Scientific American article in February 1978 (Costerton, J. W., Geesey, G. G. \& Cheng, K. J. (1978) How bacteria stick. Scientific American 238, 86-95) was that these surface associations were the rule (and not the exception) in all nutrient-sufficient microbial ecosystems, and that most bacteria in the biosphere grow in biofilms.

During the 1980s biofilms were studied because they presented anomalies and problems that could not be understood using the concept of bacteria as predominantly planktonic cells, or solved using methods that were developed for the study of individual floating organisms. Amongst these problems were misleading data on substrate turnover in rivers when extrapolated from the activities of planktonic populations - the real rates were orders of magnitude higher. Another problem involved convoluted arguments used to explain how bacterial cells floating past metal surfaces could mediate the damaging corrosion processes that cost industry huge sums of money in microbially influenced corrosion. These arguments simply dropped away when it was shown that such corrosion is caused by bacteria growing in biofilms and changing the redox state of the metals with which they are in direct and sustained contact. The inherent resistance of industrial biofilms to biocides was explained by the biofilm concept, as was the efficacy of the physical removal techniques (scraping and "pigging") that have always been effective in corrosion control. As a part of this understanding of surface-associated bacteria, the beneficial process of cellulose digestion and the deleterious processes of biodegradation were both rationalized in terms of the concentration of enzymes immediately underneath sessile microbial communities. By the end of the decade, biofilms were expected to be found in any natural or industrial process that involved either fouling or material degradation, and were expected to be much more resistant to biocides than their planktonic counterparts grown in test tubes.
While microbial ecologists and engineers were using the biofilm concept to resolve anomalies and solve problems in natural and industrial systems, medical and dental microbiologists were beginning to apply the same concept in their systems of interest. Because the same organisms (e.g. Pseudomonas aeruginosa) are often involved in many environmental and medical problems, it was thought unlikely that they would alter their basic behaviour in colonizing any particular ecosystem, but the issue required proof. When the same methods of direct microscopy and bacterial enumeration were used in medical/dental areas, biofilms were found to be the causative agents of all device-related infections, and most other chronic bacterial infections. By the middle of the 1980s, data had been generated to show that these medical biofilms are as resistant to antibiotics as environmental biofilms are to biocides, and that these sessile communities are equally refractory to clearance by host defence mechanisms. These observations made a significant impact, because doctors had been puzzled by the fact that refractory devicerelated and chronic bacterial infections now constituted between $65 \%$ and $80 \%$ of the infections treated by physicians in the developed world. Biofilm infections have gradually replaced the acute epidemic infections caused by planktonic organisms and we are now faced with a virtual "epidemic" of low-grade chronic bacterial infections caused by organisms once thought to be nonpathogens. The basic biofilm concept explains why these infections develop slowly, why they often cause little inflammation, why they often yield negative cultures, why they resist host defenses, and why they respond poorly to antibiotic therapy. By the mid-1990s biofilms had been found to be involved in so many chronic infections that biofilm microbiologists could assess the aetiology and progress of any given infection and predict the course of the infection and the efficacy of traditional therapeutic strategies. Once again, the biofilm concept had provided the intellectual framework for the resolution of anomalies and the solution of problems, and the characteristics of medical biofilms had not proved to be different from those of biofilms in other ecosystems.

It was the imperative of industrial and medical anomalies and problems that first drew practical microbiologists to the biofilm concept, and this interest attracted more than 650 attendees to the "Biofilms 2003" meeting held recently in Victoria, British Columbia. Because microbiology is divided into anthrocentric disciplines (industrial/medical/agricultural microbiology, etc.), the natural tendency of bacteria to live in biofilms has affected each discipline (in turn), until most microbiologists are now aware of the biofilm concept. But the real impact of the biofilm concept on microbiology will be much more profound than the mere resolution 
of anomalies and the solution of practical problems. The biofilm concept states that bacteria have the capability of forming complex integrated communities, and that the most highly differentiated form of bacterial life is not the planktonic cell we have studied since the time of Robert Koch, but it is the multi-species biofilm. To cast the issue in modern molecular terms, we know all about $P$. aeruginosa genes that control flagellar motility or chemotaxis, but we know virtually nothing of genes that control community structure or metabolic cooperation with other species in biofilms.

What do we know about the biofilms that constitute the predominant mode of growth of bacteria in the biosphere? In the early 1980s we knew that $99.9 \%$ of bacteria in aquatic ecosystems lived on surfaces, and some researchers suggested that the organisms might be moribund, but we found that they were also predominant in metabolic activity. Even in the late 1980s, we thought that biofilms were simply random accretions of cells in matrix material, but the early 1990s and the scanning confocal laser microscope brought the realization that most biofilms are composed of structured microcolonies and ramifying water channels (Fig. 1). Direct observations of living biofilms showed that the microcolonies were composed of matrix material $( \pm 85 \%)$, and cells $( \pm 15 \%)$, and exhibit the properties of a viscoelastic fluid. Both microscopy and nuclear magnetic resonance revealed that the water channels accommodate convective flow, driven by the flow of the bulk liquid, so that the sessile community is served by a de facto "circulatory system". Observations of natural biofilms, ranging from dental plaque to accretions in sewage treatment plants, show that the basic microcolony/water channel pattern is seen in most sessile communities, and that strong shear forces may deform the microcolonies to produce oscillating streamers. When the tensile strength of the microcolonies is exceeded, large fragments leave the biofilm, and stagnant conditions trigger the release of planktonic cells from sessile populations, so that these communities tend to propagate downstream. Now that we have direct methods to visualize biofilms, and we have probes for cell viability and species identity, there seems to be no reason not to use confocal microscopy to determine the structural organization of biofilms in all systems of interest. We really need to know what an organism looks like if we are to study it in a meaningful way, and the "organisms" that microbiologists study are the biofilms formed by single or multiple species of bacteria on surfaces in various ecosystems.

The most profound consequence of the biofilm concept, which is also the direct demonstration that sessile communities have a structure in which each cell has a different microniche (and phenotype), is the deduction that these communities must have some system of cell-cell communication. Dozens of recent papers have shown that certain cell-cell signals are essential for biofilm formation, and five or six signalling systems have been implicated, but these broad biofilm control systems are of less interest than the more subtle signals that control community structure. The search for the specific signals that control such structural features as the maintenance of water channel integrity will last for decades and involve signal systems that have yet to be discovered. Biofilms contain cells in almost every physiological state known to science, from aerobic to strictly anaerobic and from "exponential" to "stationary" phase of growth, and the analysis of gene expression in biofilms shows a vast increase in expression over that seen in any liquid culture. More importantly, the analysis of gene expression in biofilms shows that genes controlling non-metabolic cellular properties (e.g. porins or membrane pumps) are upregulated in biofilms, indicating the existence of a specific biofilm phenotype. In an even more tantalizing preliminary study, it has been shown that cultivation in a biofilm structurally juxtaposed to the biofilm of another species upregulates a whole set of genes, most of which have never been seen to be expressed in single-species cultures. We predict that the microscopic exploration of gene expression in biofilms, using reporter constructs, will show a sequence of gene activation not unlike that of the sequence seen in the embryology of higher multicellular organisms. Our challenge will then be to find the cell-cell signals that control this developmental process, in the same way that hormones control the ontology of plants and animals. Bacterial biofilms will, however, always have the unique property of being derived from the controlled interaction of the genomes of more than one species, which can be mobilized to make a mature organism that can rapidly take advantage of nutrient opportunities.

This new journal is launched at a very opportune moment. As the essential unity of the structure and function of biofilms is recognized, isolated observations of sessile communities in very different ecosystems can be applied to biofilms in general. If certain internal cells in biofilms on plant roots are sufficiently anaerobic to carry out nitrogen fixation, then we might look for areas in the biofilms in the lungs of patients with cystic fibrosis that are sufficiently anaerobic to use nitrate (instead of oxygen) as an electron acceptor. If it is proposed that alterations in porin composition make biofilm cells of certain bacteria much more resistant to traditional antibiotics, then the analysis of gene expression in sessile populations should show the upregulation of porin genes in sessile communities. In essence, a matrix of biofilmrelated data has been generated during the past two decades, at a sharply increasing rate, and new data are especially useful if they are published in a format in which they can readily be integrated into the whole biofilm picture. In some cases, we may not know exactly where each piece fits into the puzzle but, as time passes and the big picture emerges, a fact or observation that initially seems isolated may slide in to a critical place and contribute to a concept of pivotal importance to modern microbiology.

The aims of this new journal are to provide a single forum for the publication of articles relevant to biofilms and to be the journal of choice for researchers in this very broad field. It will be interdisciplinary and multidisciplinary and will encompass all of the subject areas mentioned above. Biofilms, therefore, welcomes manuscripts from microbiologists, engineers, clinicians, 
ecologists, microscopists, mathematicians, marine biologists, chemists and others working on biofilms that encompass the best research currently being undertaken so that the journal will serve to pioneer progress in the field. A principal objective is to ensure that new and original research is published within six months of submission so that as the journal grows we will produce issues more frequently rather than simply increasing page numbers. We also intend to be responsive to the needs of our readers and so welcome your views on this issue (and subsequent issues) of the journal and are always willing to consider suggestions regarding ways in which we can be of value to the biofilm community. 